\title{
The Role of Diffusion Tensor Imaging (DTI) in Developmental Brain Anomalies in Pediatric Age Group
}

\author{
DINA H. FAWZY, M.Sc.; HADEEL M. SEIF EL-DIEN, M.D. and RANIA Z. HASSEN, M.D. \\ The Department of Radiology, Faculty of Medicine, Cairo University
}

\begin{abstract}
Background: Neurodevelopmental disorders are complex processes with underlying abnormalities of white matter tracts. DTI is an advanced neuroimaging technique that can delineate the underlying abnormal white matter tracts in these disorders.

Purpose: To demonstrate the role of diffusion tensor imaging and the tarctography techniques in the diagnosis of brain developmental anomalies in pediatric age group.

Patients and Methods: This is a prospective study carried out in Radio-Diagnosis Department, Faculty of Medicine, Cairo University between March 2014 and October 2015. It included eleven patients ( 1 day-15 years) having neurological dysfunctional symptoms and ten control subjects of the same age group. Conventional MRI images and tractography maps were used. The examined tracts were Corpus Callosum (CC), corticospinal tract (Cst), superior and inferior longitudinal fasciculi (SLF, ILF). Fractional Anisotropy (FA) was measured at bilateral frontal, parietal and occipital white matters regions.

Results: Nine cases out of eleven cases with positive conventional MRI findings showed additional DTI findings. e.g tract disruption, absent or attenuated fibers Altered FA values in the white matter tracts were also found.

Conclusion: This study emphasis the feasibility of applying DTI technique in the evaluation of developmental brain anomalies to demonstrate additional findings not clearly visualized in conventional MR images to increase the diagnostic accuracy.
\end{abstract}

Key Words: Diffusion tractography - Echo-Planar Imaging (EPI) - Nervous system malformations - Metabolic - Neurodevelopmental disorders.

\section{Introduction}

NEURODEVELOPMENTAL brain abnormalities are known to be of variable and complicated nature. Diagnosing such abnormalities using neuroradiological techniques, needs considerable basic awareness of both normal and pathogenic brain development [1].

Correspondence to: Dr. Rania Z. Hassen, E-Mail: dr raniaz@hotmail.com
Imaging has a vital role to form the accurate diagnosis required for optimum management of these conditions [1].

When compared to MRI, Diffusion Tensor Imaging (DTI) and Fiber Tractography (FT) have shown better ability to characterize aberrant white matter connections that are frequently revealed in developmental brain anomalies and white matter dysmyelination [2].

DTI is a recent non-invasive technique that provides the ability to investigate water diffusion in the cerebral White Matter (WM) in better detail, through revealing the direction of water diffusion in the WM and quantifying its magnitude [3]

\section{Patients and Methods}

This is a prospective study carried out in RadioDiagnosis Department, Faculty of Medicine, Cairo University between March 2014 and October 2015. The study included 11 patients having motor or developmental delay, (between 1 day and 15 years of age) referred from (Neurology Outpatient Clinic, Pediatric Hospital, Cairo University Hospitals), and 10 control subjects of the same age group to get reference measurements for white matter FA values and anatomical tracts configuration.

Our institutional review board waived the requirement of informed consent; however normal controls subjected to informed consent according to the ethical committee regulations.

Inclusion criteria: From neonate to 15 years old patients having neurological dysfunction Ten control subjects of the same age group.

Exclusion criteria: General: Claustrophobic patients, patients with pacemaker or metallic im- 
plants. Specific: Patients suffering from ischemic or traumatic brain injuries.

MR examination included: Non contrast Conventional MR examination including T1WI, T2WI, and FLAIR (fluid attenuation inversion recovery) in axial, sagittal and coronal planes. Diffusion Tensor imaging medium.

The technique was performed using a standard 1.5 Tesla unit, pediatric head coil was used in supine position, and twenty cases were under sedation. Scan time was around 12 minutes.

\section{Imaging parameters:}

FLAIR: TR/TI 6000/2000ms, TE 120ms, matrix 208 X 192, FOV 210 X 236mm, number of excitation: 2 , slice thickness: $6.0 / 1.5$.

Diffusion Weighted Imaging (DWI): A diffusion weighting factor of $\mathrm{b}$ zero and $1000 \mathrm{~s} / \mathrm{mm}^{2}, \mathrm{TR}$

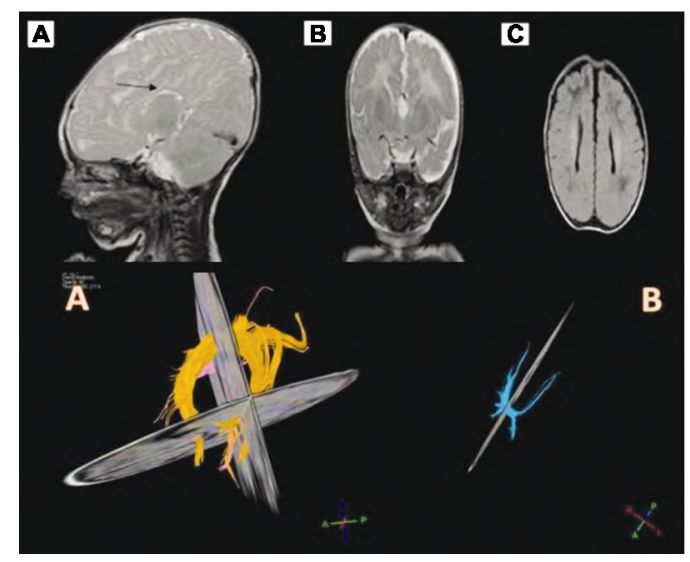

Fig. (1): 5 month old male presented with metabolic acidosis and respiratory distress grade two. Conventional MRI sagittal, coronal T2WI showing hyper-intense signal in white matter frontal region, non-visualized $\mathrm{CC}$ [arrow] $(\mathrm{A}, \mathrm{B})$, axial FLAIR showing parallel frontal horns of lateral ventricles (C). 3DDTI reconstructed image, showing the probst bundles on both sides, $\mathrm{H}$ shaped fibers connected at the region of the genu of corpus callosum.

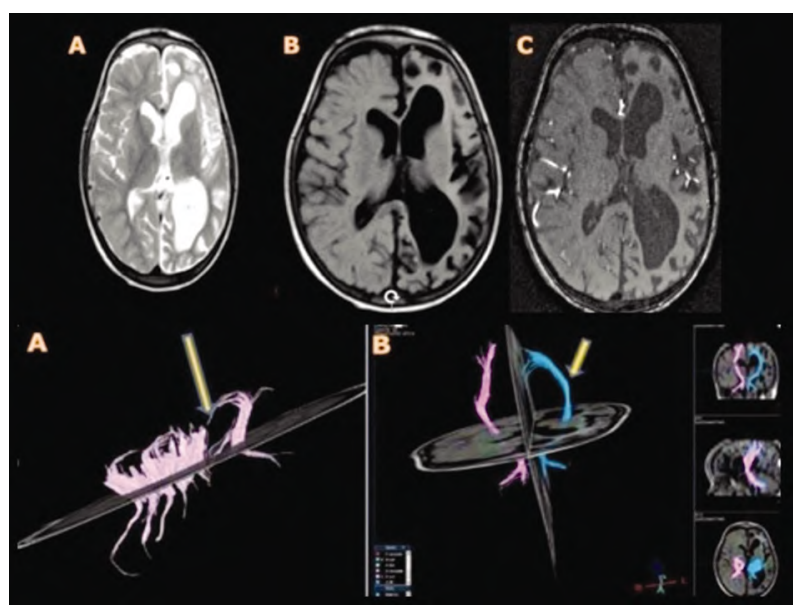

4100ms, TE $115 \mathrm{~ms}, 90^{\circ}$, matrix 132 X 105, FOV $210 \times 236 \mathrm{~mm}$, number of excitation: 1 , slice thickness: $6.0 / 1.5$.

Diffusion tensor consisted of: A single shot, spin-echo echo planar sequence in 16 encoding directions, a diffusion weighting factor of 800 $\mathrm{s} / \mathrm{mm}^{2}$, TR $8000 \mathrm{~ms}$, TE $67 \mathrm{~ms}$, flip $90^{\circ}$, matrix 112 X 110, FOV 210 X 236mm, number of excitations: 2, slice thickness: 2.0/00.

\section{Results}

Table (1) showed comparison between the diagnosis by conventional MRI and DTI findings.

Nine cases out of eleven cases with positive conventional MRI findings showed additional DTI findings. Combined structural and anatomical disorders were noted in five out of eleven cases.

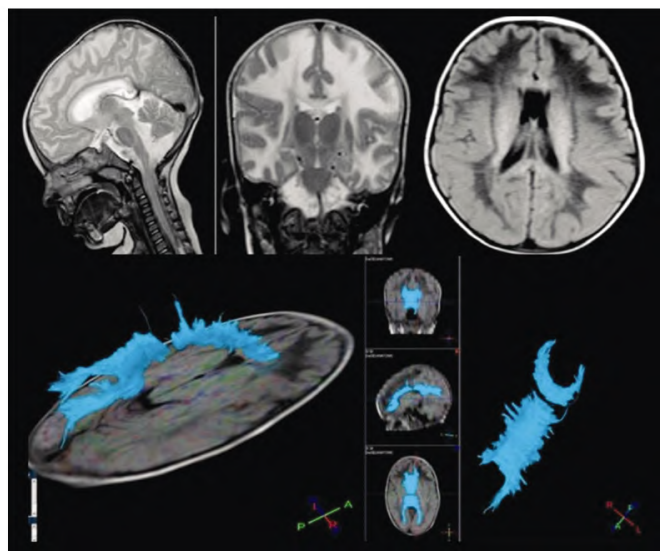

Fig. (2): One and half year old male patient suffering from generalized hypotonia and delayed mile stones. Conventional MRI sagittal and coronal T2WI showing the corpus callosum and diffuse hyperintense signal. Axial FLAIR showing diffuse hypointense signal. 3DDTTI showing centrally interrupted callosal fibers.

Fig. (3): 5 year old male presented with unilateral intractable seizures Conventional MRI axial T2WI (A), axial FLAIR (B) and T1 with contrast images (C); showing unilateral cortical atrophy with ex-vacuo ventricular dilatation, hyperintense signal in T2WI and FLAIR in the left hemisphere, T1 $\mathrm{C}+(\mathrm{Gd})$-no significant post-contrast enhancement. 3D DTTI the CC fibers are disrupted posteriorly [arrow] (left image) and corticospinal tracts show marked displacement on the left side [arrow] right. 
Table (1): Comparison between the diagnosis by conventional MRI and DTI findings.

\begin{tabular}{|c|c|c|c|}
\hline Case $\mathrm{N}$ & Diagnosis (conventional MRI) & Anatomical by DTI & Functional by DTI (FA) \\
\hline Case 1 & - Total ACC. & - Partial ACC. & - Periventricular low FA. \\
\hline Case 2 & - Joubert dandy variant. & - Attenuated, splayed SCP. & $\begin{array}{l}\text { - No regional white matter or tracts } \\
\text { abnormality. }\end{array}$ \\
\hline Case 3 & $\begin{array}{l}\text { - Polymicrogyra, cortical dysplasia } \\
\text { (Zellwegar syndrome). }\end{array}$ & - Thick CC and distal fiber crossing. & $\begin{array}{l}\text { - Decreased FA in frontal and parietal } \\
\text { regions. }\end{array}$ \\
\hline Case 4 & - Pachygyria, cortical dysplasia. & $\begin{array}{l}\text { - Deviated tracts at the dysplastic grey } \\
\text { matter. }\end{array}$ & $\begin{array}{l}\text { - No regional white matter or tracts } \\
\text { abnormality. }\end{array}$ \\
\hline Case 5 & - Subependymal heterotopia. & • No tracts abnormality. & - High FA in heterotopic grey matter. \\
\hline Case 6 & $\begin{array}{l}\text { - Cavum valum interposial cyst (cyst } \\
\text { displacing white matter). }\end{array}$ & - Displacement of the left CST. & $\begin{array}{l}\text { - No regional white matter or tracts } \\
\text { abnormality. }\end{array}$ \\
\hline Case 7 & - Total ACC. & - Total ACC, with bilateral probst bundles. & $\begin{array}{l}\text { - No regional white matter or tracts } \\
\text { abnormality. }\end{array}$ \\
\hline Case 8 & • Adrenoleukodystrophy. & - Defective CC fibers centrally. & $\begin{array}{l}\text { - Diffuse decrease in regional white matter } \\
\text { FA. }\end{array}$ \\
\hline Case 9 & - Leukodystrophy. & $\begin{array}{l}\text { - Complete anatomical tract disruption, } \\
\text { (single ROI). }\end{array}$ & $\begin{array}{l}\text { - Diffuse decrease in regional white matter } \\
\text { FA. }\end{array}$ \\
\hline Case 10 & - Rasmussen encephalitis. & - Defective CC fibers posteriorly. & $\begin{array}{l}\text { - Unilateral decrease in the regional white } \\
\text { matter FA. }\end{array}$ \\
\hline Case 11 & - Canavan. & - No tracts abnormality. & $\begin{array}{l}\text { - Diffuse decrease in regional white matter } \\
\text { FA? }\end{array}$ \\
\hline
\end{tabular}

\section{Discussion}

DTI has shown remarkable results concerning the studies of variable developmental brain disorders [4]

In cases showing negative conventional MRI studies, surveying the entire brain through sensitive screening techniques is becoming more useful. Using the DTI technique to measure the anisotropy can help to detect hidden anomalies, as it is more sensitive, compared to other contrast mechanisms [5].

The corpus callosum starts to develop at the genu portion followed by the body, the splenium, and lastly the rostrum. Therefore, the posterior part or rostrum is hypoplastic in the case of partial Agenesis of the Corpus Callosum (ACC) [2] .

The focus was specifically on the $\mathrm{CC}$, because it provides immense interhemispheric connections between the corticospinal and the homologous neocortical areas. This plays a significant role in memory, attention, sensorimotor integration and the general cognitive functioning [3]

A case was reported in this study by conventional MRI as complete corpus callosum agenesis with associated increased white matter signal in T2WI, DTI-FT showed that it was a partial callosal agenesis. Fig. (1). There was sparse fibers at the region of the genu taking $\mathrm{H}$ shaped pattern Fig. (1) these findings are also described by [6], together with increased FA at the region of the genu and reduction of the FA in the frontal region.
Agenesis of corpus callosum is characterized by typical MR imaging findings such as a "cartwheel configuration" of the interhemisphericsulcal markings, absence of the cingulate gyrus, and colpocephalic features of the lateral ventricles [2]

A case was reported in this study by conventional MRI as corpus callosum agenesis, the diagnosis was confirmed by the DTI-FT as complete callosum a genesis and the misdirected antroposterior probst bundle, in an attempt to connect both hemispheres was shown this coincides [2].

At DTI-FT, the fibers from the hemispheric cortex, did not cross the midline but they form a thick bundle in the anteroposterior region (Probstbundle) [2].

Such fibers are probably formed from misdirected callosal axons and are of unknown functions but they commonly appear in the subjects with Agcc [7].

Joubert syndrome is known to be a subtype of the malformation of posterior fossa. It is manifested as vermian hypoplasia and derangement of the cerebellar-brainstem or cerebello-cortical connections. "Molar tooth appearance" of the Superior Cerebellar Peduncle (SCP) together with partial or complete absence of the vermis are demonstrated at MR imaging. Meanwhile, elongated SCP with a horizontal configuration can be seen at DTI and FT techniques. The detailed description of the fiber was impossible using conventional imaging [2] . 
It is here worth mentioning that, DTI and FT are the only methods that can demonstrate these aberrant fiber connections in the diseased state. This surely provides a better understanding of a malformed brain [2]

A case was reported in this study by conventional MRI as Joubert-dandy complex with mid brain typical molar tooth appearance, by the DTIFT the SCP was thin attenuated which was described Lee et al., [2].

A case was reported in this study with conventional MRI as a large CSF filled inter-hemispheric cyst, seen behind the foramen of Monro and have a triangular configuration pointing anteriorly, on coronal and axial imaging the fornixes are elevated and splayed, by DTI-FT the lower part of the CST and superior cerebellar peduncle was splayed around the cyst especially on the left side by virtue of its mass effect.

DTI and FT are useful in evaluating the integrity of the WM adjacent to dysplastic cortex. Moreover, in cases of severly dysplastic white matter, DTIFT can detect the aberrant course of white matter tract [2].

A case was reported in this study by conventional MRI with pachygyrii and cortical dysplasia. DTI-FT shows disruption of the callosal fibers posteriorly and at the defective sulcation around the dysplasia, as described by Lee et al., [2] .

In case of heterotopic grey matter, arrested neurons that might have some directivity like the normal white matter tracts appear in the white matter bundles, showing high anisotropy. Meanwhile, grey matter in the WM which is nodular or forming band heterotopia, demonstrated significantly higher anisotropic value as compared to the normal cortex [2]

A case was reported in this study by conventional MRI as diffuse periventricular nodular impressions seen affecting the ependyma of the bodies of both lateral ventricles, which have the same signal as grey matter suggesting; "subependymal periventricular diffuse nodular heterotopia" by DTI-FT there was no significant extra information apart from the increased FA in the heterotopic grey matter than the normal grey matter which coincides by the radial migration of the grey matter described by Lee et al., [2].

A case was reported by conventional MRI as diffuse non uniform sulcal arrangement "polymicrogyra" with multiple bilateral periventricular white matter signal alteration, hyper-intense in $\mathrm{T} 2$ and FLAIR images with vacuolated appearance proven Zellweger syndrome. By DTI-FT there was thick callosal fibers and crossing heart shaped corticospinal tract cranially, associated with mild decrease in the FA of the frontal and parietal regions.

DTI has a powerful ability to demonstrate the integrity of the white matter and allows detection of abnormalities of the brain tissue in an earlier stage than conventional T2-or T1-weighted imaging [2].

Patients suffering from some cases of mitochondrial disease can remarkably benefit from DTI-FT studies. Such cases are characterized by sponginess, myelin deficient formation and loss, rich amounts of lipid-laden macrophages, capillary proliferation, prominent gliosis and eventually axonal loss [7].

Moreover, quantitative DTI parameters can also help detecting structural changes in cases of dysmyelination, especially those who did not show WM abnormalities on conventional MRI [7]

A case was reported in this study by conventional MRI and complementary MRS that show diffuse white matter T2 hyper intensity in the deep and periventricular and subcortical fibers Fig. (2) (MRS revealed large lactate peak small MI peak ;no reduction of NAA, no increase of choline), picture suggesting leukodystrophy and proven to be adrenoleukodystrophy, by DTI-FT there was interruption of callosal fibers in its central portion Fig. (2) and non-visualized SLFs; the FA were reduced on both sides in frontal parietal and occipital regions coinciding with the axonal loss described by Ishak et al., [7].

A case was reported by conventional MRI with Bilateral diffuse altered signal intensity of both deep white matter; bright in T2WI and dark in T1WI and FLAIR, sparing deep grey matter i.e. both basal ganglia and thalami and lateral capsule suggesting leukodystrophy, DTI-FT showed complete anatomical disruption of all white matter tracts as described by axonal loss theory by Ishak et al., [7] together with global decrease of FA in frontal, parietal and occipital region.

A case was reported at the current study by conventional MRI as-unilateral hemispheric parenchymal atrophy with ex-vacuo ventricular dilatation, likely post inflammatory. diffuse white matter, T2-hyper intense signal areas were seen (Rasmussen encephalitis) Fig. (3), DWI images revealedrestricted diffusion at the altered signal areas, MRA of this patient was free by DTI-FT there was de- 
fective callosal fibers posteriorly and shift of the Cst to the left in addition to significant decrease of FA on the left side Fig. (3) coinciding with axonal loss as described by Ishak et al., [7].

A case was reported in this study by the conventional MRI and complementary MRS showing diffuse bilateral symmetrical cerebral and cerebellar areas of white matter abnormal signal eliciting bright T2WI, low in T1WI and FLAIR and involving both deep and subcortical U fibers, bilateral abnormal signal of deep grey matter relatively sparing the putamina with total involvement of the brain stem were also seen. MRS shows increased NAA peak, MI peak, small GLs and small lactate. "Canavan disease", by DTI-FT there was diffuse decrease of the FA of the frontal, parietal and occipital regions as suggested by Ishak et al., [7]

In conclusion, DTI technique now makes it possible to accurately show the anatomy of the white matter, and to precisely detect neurological abnormalities in pediatric symptomizing patients whose conventional MRI studies cannot clearly point out their disorder. In the field of pediatric radiology, DTI can become a vital tool for studying the brain anatomy, not only for the diagnosis of various white matter-related disorders, but also in the prognosis and assessment to treating such disorder.

\section{Acknowledgment}

We would like to express our gratitude to the European society of radiology where this work was firstly presented in the 5th of March 2016 in the European congress of radiology (ECR) as an electronic poster with oral presentation in the new voice of EPOS session.

Sincere thanks Dr. Ayman Abd El-Hamid AlBasmy lecturer of Radiology, Cairo University for his support and continuous guiding.

\section{References}

1- BANO S., CHAUDHARY V. and YADAV S.: Congenital malformation of the brain, Neuroimaging-Clinical Applications, Prof. Peter Bright (Ed.), InTech, doi: 10.5772/ 22936, 2012.

2- LEE S., KIM D., KIM J., et al.: Diffusion-tensor MR imaging and fiber tractography: A new method of describing aberrant fiber connections in developmental CNS anomalies. Radiographics, 25: 53-65, 2005.

3- CANCELLIERE A., MANGANO F., AIR E., et al.: DTI values in key white matter tracts from infancy through adolescence. Am. J. Neuroradiol., 34: 1443-9, 2013.

4- HOON A.H. Jr., LAWRIE W.T. Jr., MELHEM E.R., et al.: Diffusion tensor imaging of periventricular leukomalacia shows affected sensory cortex white matter pathways. Neurology, 59: 752-6, 2002.

5- MORI S. and TOURNIER J.: Introduction to diffusion tensor imaging and higher order models. 2 nd ed. Elsevier, 2014.

6- PFEFFERBAUM A., SULLIVAN E.V., HEDEHUS M., et al.: Age-related decline in brain white matter anisotropy measured with spatially corrected echo-planar diffusion tensor imaging. Magnetic Resonance in Medicine, 44: $259-68,2000$

7- ISHAK G.E., POLIAKOV A.V., POLIACHIK S.L., et al.: Tract-based spatial statistical analysis of diffusion tensor imaging in pediatric patients with mitochondrial disease. A.J.N.R., 33 (9): 1726-30, 2012.

\section{دور الرنين المغناطيسى الإنتشارى الموتر للآلياف العصبية

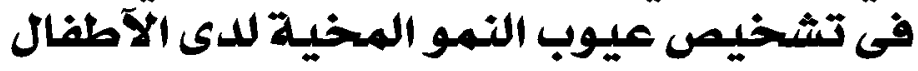

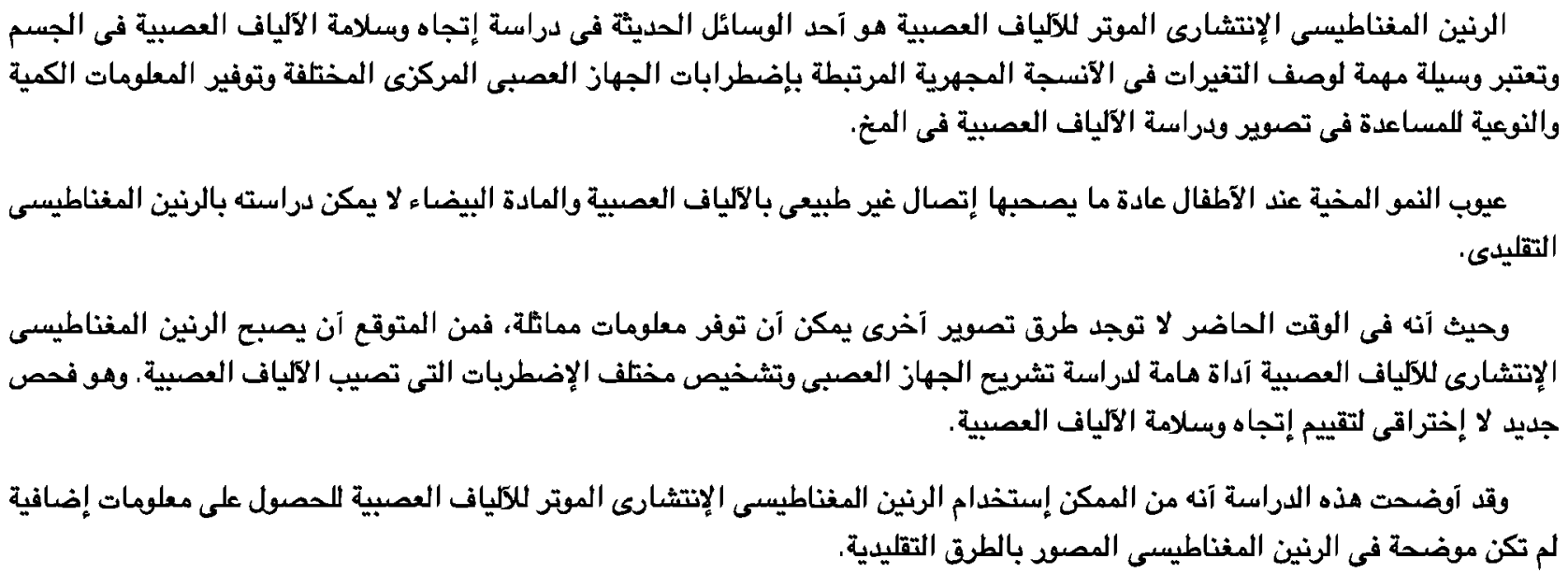

